\title{
ARTICLE MICU1 may be a promising intervention target for gut-derived sepsis induced by intra-abdominal hypertension
}

\author{
Yuxin Leng, Qinggang Ge, Zhiling Zhao, Kun Wang and Gaiqi Yao
}

Intra-abdominal hypertension (IAH) is a common and serious complication in critically ill patients, for which there is no targeted therapy. IAH-induced dysfunction of intestinal barriers is closely associated with oxidative imbalances, which are considered to provide a pathophysiological basis for subsequent gut-derived sepsis. However, the upstream mechanism that produces oxidative damage during IAH remains unknown. It is not clear whether 'mitochondrial $\mathrm{Ca}^{2+}$ uptake $1^{\text {' }}$ (MICU1, the key protein regulating the oxidative process) is involved in preventing $\mathrm{Ca}_{\mathrm{m}}^{2+}$ (mitochondrial $\mathrm{Ca}^{2+}$ ) overload. Here, we detected changes in the expression of MICU1 during the development of increased intestinal permeability in rats with $\mathrm{IAH}$, and we explored the related mechanism regulating epithelial-barrier functions by knocking-down micul in Caco-2 cells. Our results demonstrated that, to combat IAHinduced dysfunction of intestinal barriers, MICU1 undergoes a compensatory increase in expression, whereas 'mitochondrial calcium uniporter' (MCU) - a conserved $\mathrm{Ca}^{2+}$ transporter - becomes transcriptionally suppressed. Silencing the expression of MICU1 destroyed Caco-2 cell barrier integrity, promoted paracellular permeability, and impaired the expression of tight junction proteins (occludin, ZO-1, and claudin 1). Meanwhile, oxidative imbalances were induced; malondialdehyde (MDA), a product of oxidation, was increased and antioxidant products (GSH-Px, CAT, and SOD) were decreased. In MICU1-deficient Caco-2 cells, proliferation was inhibited and apoptosis was promoted. Collectively, our results indicate that MICU1-related oxidation/antioxidation disequilibrium is strongly involved in IAH-induced damage to intestinal barriers. MICU1-targeted treatment may hold promise for preventing the progression of IAH to gut-derived sepsis.

Cell Death Discovery (2016) 2, 16080; doi:10.1038/cddiscovery.2016.80; published online 28 November 2016

\section{INTRODUCTION}

Intra-abdominal hypertension (IAH) is a sustained or repeated increase in intra-abdominal pressure (IAP), greater than or equal to $12 \mathrm{mmHg}$ (10 mmHg for children), without well-defined intervention strategies. ${ }^{1}$ Intestinal barrier functions have been confirmed to be significantly damaged during $\mathrm{IAH}$, which may trigger gutderived sepsis and multi-organ failure (MOF). ${ }^{2-5}$ Oxidative stress following $\mathrm{IAH}$-induced ischemia has been implicated as the mechanism underlying the damage. It has been reported that the elevation in IAP causes disturbances in the intestinal microcirculation, which are followed by enhanced oxidant responses and bacterial translocation. ${ }^{5-8}$ The balance between oxidative stress and antioxidation processes can be destroyed even before the level of IAP matches the level in the diagnostic criteria. ${ }^{9}$ However, the upstream mechanism underlying the oxidative imbalance induced by increased intestinal permeability remains unclear.

Mitochondrial $\mathrm{Ca}^{2+}\left(\mathrm{Ca}_{\mathrm{m}}^{2+}\right)$ homeostasis is critical for maintaining normal cellular physiology. Under normal conditions, the $\mathrm{Ca}^{2+}$ concentration in the mitochondrial matrix is maintained 5-6 orders of magnitude lower than its equilibrium level. 'Mitochondrial $\mathrm{Ca}^{2+}$ uptake 1' (MICU1), which is an EF hand $\mathrm{Ca}^{2+}$ binding protein, monitors mitochondrial $\mathrm{Ca}^{2+}$ levels and sets a $\mathrm{Ca}^{2+}$ threshold for $\mathrm{Ca}_{\mathrm{m}}^{2+}$ uptake, mediated by 'mitochondrial calcium uniporter' (MCU). Therefore, MICU1 prevents $\mathrm{Ca}^{2+} \mathrm{m}$ overload and the associated oxidative cellular damage. ${ }^{10,11}$ The regulatory function of MICU1, namely preventing oxidative damage, has been confirmed in HeLa cells, hepatocytes, and endothelial cells. It has been reported that, in the absence of MICU1, excessive MCUmediated $\mathrm{Ca}^{2+}$ uptake leads to overproduction of mitochondrial reactive oxygen species (mROS) and sensitivity to apoptotic stimuli. $^{10-12}$

Accordingly, considering the close connections between MICU1, oxidative stress, and IAH-induced intestinal barrier dysfunction, it is reasonable to hypothesize that dysfunction of MICU1 is the upstream mechanism for IAH-related mucosal barrier damage. In the present study, we aimed to investigate the $\mathrm{IAH}$-induced changes in the expression of MICU1 and MCU in rats. In addition, we tried to verify the importance of MICU1 in maintaining the integrity of the intestinal epithelium, using genetic knockdown.

\section{RESULTS}

A 90-min exposure to IAH elicited increased intestinal permeability We determined gut permeability to macromolecules by measuring FD-4 leakage from the gut cavity into the portal circulation. As shown in Figure 1, similar to our previous findings, a 90-min exposure to IAH resulted in a significant increase in the amount of FD-4 in the plasma of portal blood (control versus IAH: $11.1 \pm 5.9 \mu \mathrm{g} / \mathrm{ml}$ versus $158.1 \pm 16.7 \mu \mathrm{g} / \mathrm{ml}$ ), indicating that IAHinduced increased permeability models had been successfully established. 
Abnormal gene expression profiles were detected for MICU1 and $\mathrm{MCU}$ in rats with $\mathrm{IAH}$

No significant changes in the levels of MICU1 and MCU were detected in the rats with acute $\mathrm{IAH}$, using western blotting and qRT-PCR (Figure 2; Table 1). However, changes in the levels of MICU1 and MCU were determined at the transcriptional level (Table 1). MICU1 was significantly upregulated, whereas MCU was downregulated (Table 1). This imbalance between MICU1 and MCU, at mRNA level, during a 90 min exposure to IAH might be the result of an attempt by the cells to minimize IAH-induced damage to the intestinal barrier. Therefore, the compensatory increase in MICU1 expression suggested its importance in intestinal barrier protection.

Knockdown of MICU1 destroys the epithelial-barrier function in cultured Caco-2 cells

To elucidate the function of MICU1, we silenced its expression using four distinct RNA hairpins targeting the gene (Table 2).

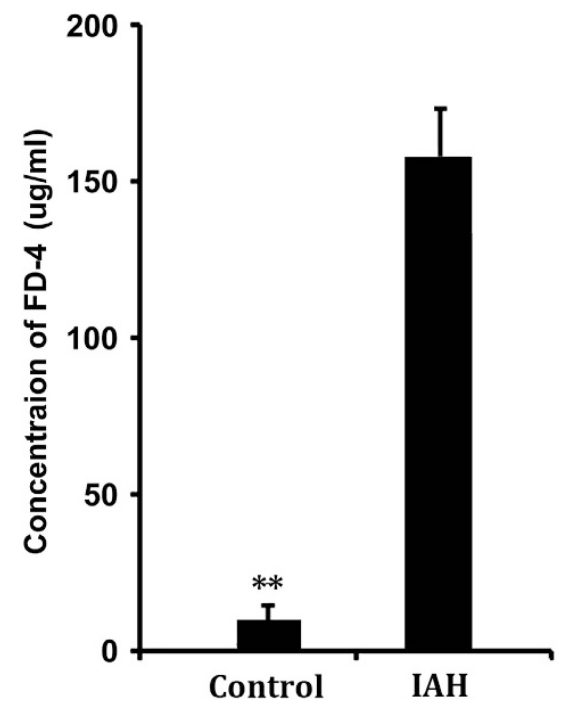

Figure 1. Establishment of IAH-induced increased intestinal permeability in rats. Ninety minutes exposure to an IAP of $20 \mathrm{mmHg}$ was determined to successfully induce increased intestinal permeability models. The concentration of FD-4 in portal blood detected was significantly higher than that of control rats. The data are shown as mean \pm S.D. values and compared by independent $t$-test ${ }^{* *} P<0.01$.
Notably, shRNA-2 was the most effective, resulting in $\sim 92.6 \%$ knockdown of MICU1 protein expression (Figure 3). All of the following results are from knockdown studies performed using shRNA-2.

Examination of tight junction proteins, the clearance of fluorescein sodium salt (FSA), and tansepithelial electrical resistance (TEER) studies demonstrated that MICU1 is essential for the protection of the mechanical barrier function of intestinal tissue in vitro (Figure 4). Formation of a Caco-2 cell monolayer was initially validated by TEER measurements. For the control Caco-2 cells, and those transfected with an empty lentiviral vector (negative shRNA control group, NC), the TEER values detected were all higher than $250 \Omega \mathrm{cm}^{2}$ at 14 days, indicating perfect compactness and integrity of their monolayers. Using these cellular monolayers, we noticed that the clearance of FSA in the MICU1-deficient group was significantly higher than that of the control group and the NC group (Figure 4a), whereas the clearance of FSA was equivalent in the control group and the NC group. The expression results for the TJs further confirmed the

Table 1. Gene expression levels of MICU1 and MCU in rats with an IAP of $20 \mathrm{mmHg}$

\begin{tabular}{|c|c|c|c|c|c|}
\hline Gene symbol & $\log F C$ & $\mathrm{t}$ & P-value & adj. P-val & B \\
\hline MICU1 & 1.529 & 6.99 & 0.0009 & 0.0738 & 0.9661 \\
\hline$M C U$ & -2.826 & -11.91 & 0.0000 & 0.0057 & 2.0278 \\
\hline
\end{tabular}

Abbreviations: logFC, a positive results indicated upregualtion; a negative results indicated downregulation; $t$, $t$-test statistic; $P$-value, unadjusted $P$-value; adj. $P$-val, adjusted $P$-value; $B$, expression index. The differential expression levels were calculated using a moderate $t$-test implemented in the Bioconductor limma package (R-statistical software) at http://www. bioconductor.org.

Table 2. Details for micu1 gene and lentiviral shRNA sense sequences

\begin{tabular}{lll}
\hline $\begin{array}{l}\text { Gene } \\
\text { (name, ID) }\end{array}$ & Ref seq & $\begin{array}{l}\text { shRNA sense sequence } \\
\text { (no. of starting base) }\end{array}$ \\
\hline micu1 10367 & NM_001195519.1 & CAGAGAAATTTTGAAATTGC (191) \\
micu1 10367 & NM_001195519.1 & GTATGCGCCACAGAGATCGT (303) \\
micu1 10367 & NM_001195519.1 & GAGAATACTGAGAGGCAGT (493) \\
micu1 10367 & NM_001195519.1 & GACATTTCAGGAGGTGGGA (610)
\end{tabular}

a

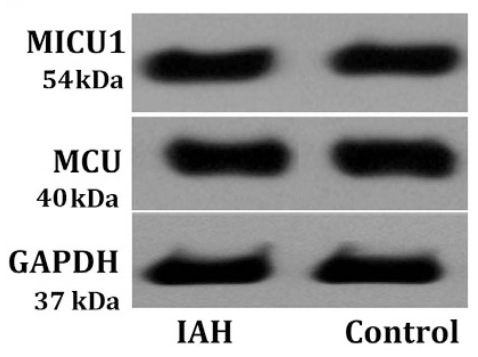

b

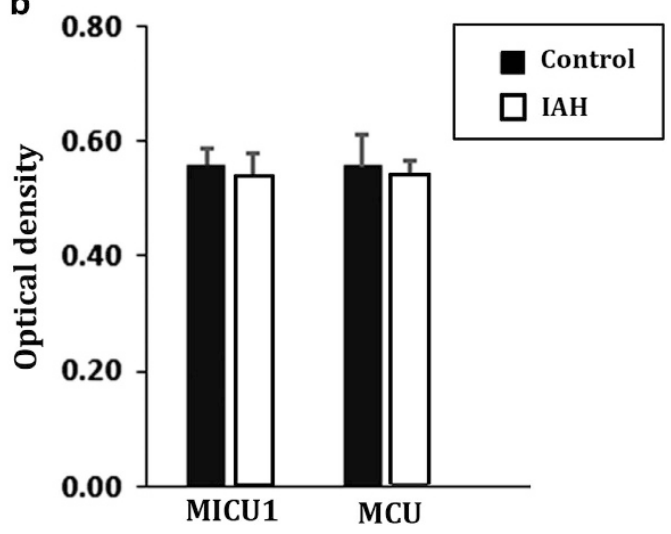

Figure 2. Expression analysis on colonic MICU1/MCU at protein level by western blotting in rats with IAH 90-min.The exposure to an IAP of 20 $\mathrm{mmHg}$ had no effect on MICU1 and MCU protein expressions. No significant differences were found between control group and IAH group. (a) The band of western blotting. (b) Analysis on gray value. The data are shown as mean \pm S.D. values and compared by independent $t$-test. 

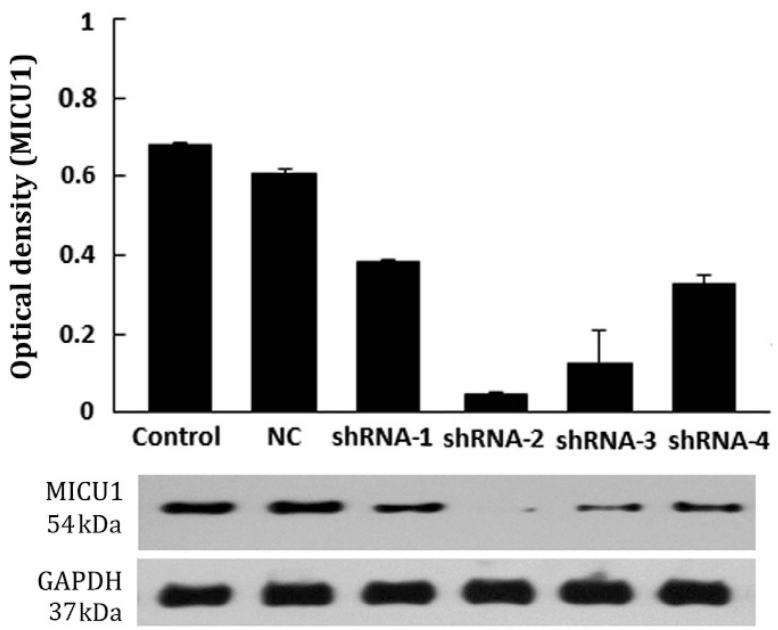

Figure 3. Identification of silencing gene micu1 in Caco-2. MICU1knockdown was identified by western blotting analysis. The four distinct hairpins (as shown in Table 2) gave 44.1, 92.6, 82.4 and $51.5 \%$ MICU1 protein knockdown, respectively. NC (negative control group), Caco-2 cells transfected with empty lentiviral vector. indispensability of MICU1 in maintaining the integrity of intestinal barriers. As shown in Figures $4 b$ and $c$, both the immunofluorescence and the western blotting analyses showed that the levels of claudin 1, occludin, and ZO-1 - which are responsible for TJ permeability and paracellular transport - were all markedly reduced when MICU1 was silenced.

MICU1 dysfunction is the upstream mechanism underlying the oxidative disturbance that damages epithelial barriers during IAH The results of mechanistic studies have shown that MICU1 deficiency led to oxidative imbalances, which are closely associated with the damage to intestinal mucosa that can be induced under acute, slightly increased IAP. ${ }^{9}$ Furthermore, these studies have shown that MICU1 deficiency inhibited the growth of Caco- 2 cells and sensitized them towards apoptotic cell death. As shown in Figure $5 \mathrm{a}$, the oxidative balance became significantly disturbed in the current study, as demonstrated by increased levels of MDA - a lipid peroxidation product - and decreased antioxidant products (GSH-Px, CAT, and SOD). Meanwhile, the TUNEL assay demonstrated prominent apoptosis of colonic epithelial cells, resulting from the knockdown of MICU1 (Figure $5 b)$. The rate of apoptosis was significantly increased on

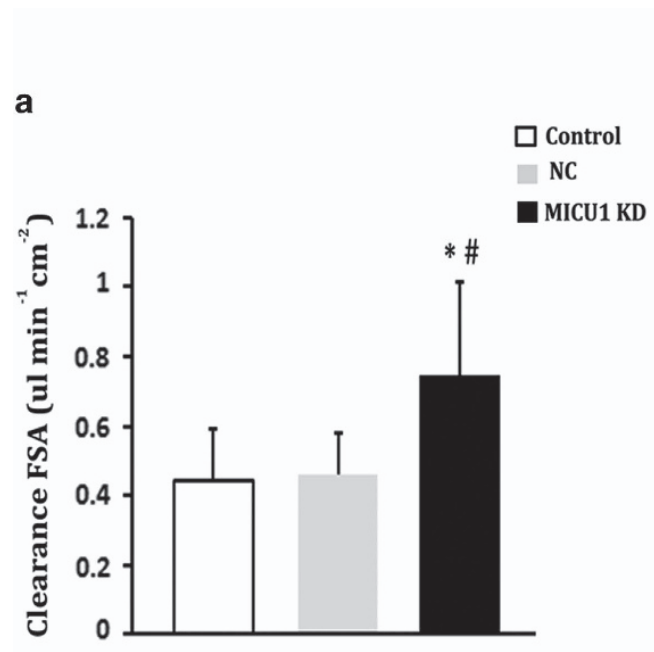

b
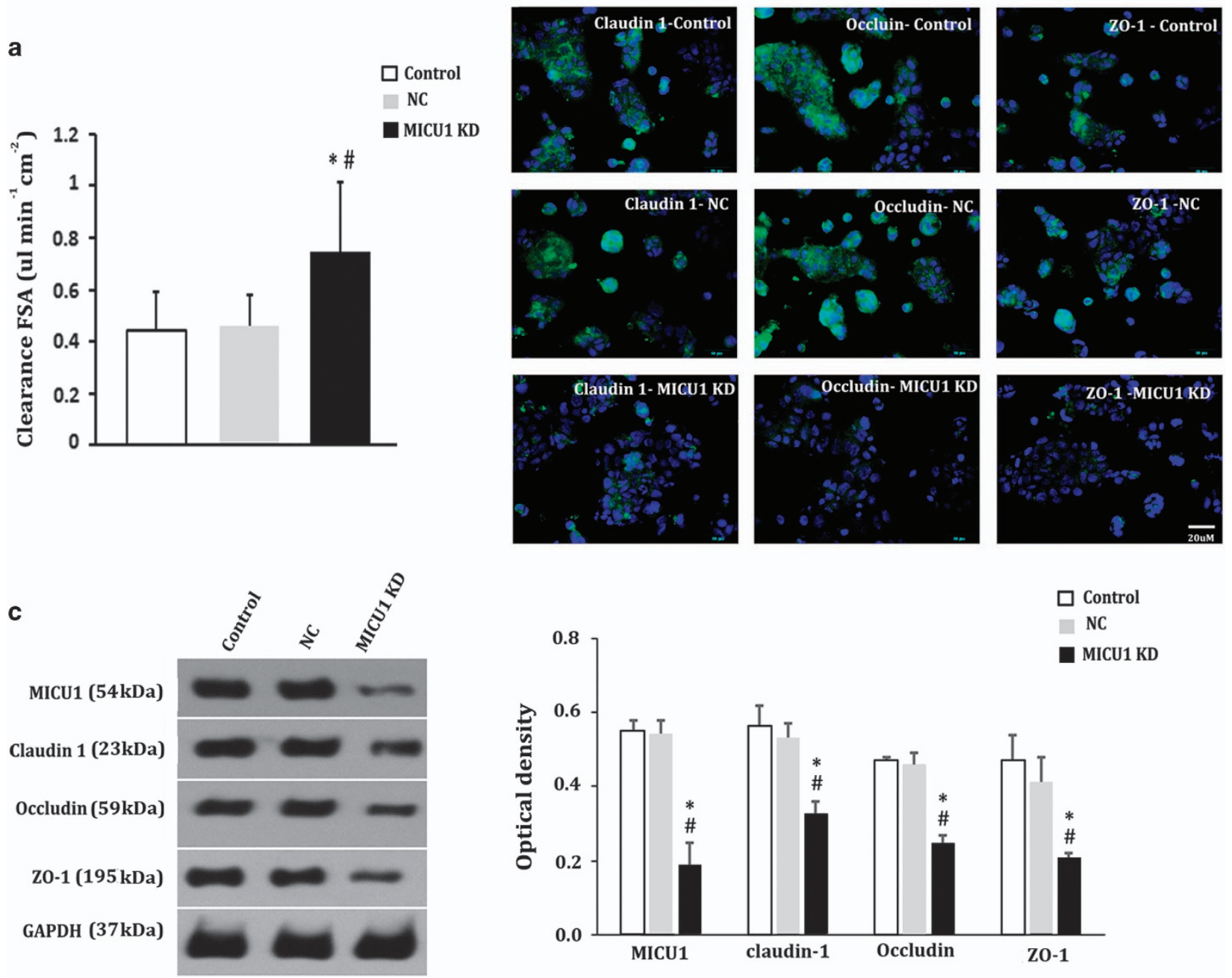

Figure 4. Involvement of MICU1 in intestinal barrier integrity in vitro. (a) Clearance of FSA. (b) Immunofluorescence analysis on tight junction proteins, claudin 1, occludin, and ZO-1 (green). (c) Western blotting analysis of tight junction proteins. Results are shown as mean \pm S.D. of three independent experiments. Statistical analysis was performed by one-way ANOVA test and independent $t$-test. versus control, ${ }^{*} P<0.05$; versus NC, ${ }^{P} P<0.05$. NC (negative control group), Caco-2 cells transfected with empty lentiviral vector. MICU1-KD, MICU1-knockdown. 
MICU1-knockdown (MICU1-KD versus control versus NC: $33.16 \pm 10.01 \%$ versus $3.64 \pm 1.33 \%$ versus $4.18 \pm 1.68 \%$; $P<0.05$ ). In addition, the MTT colorimetric assay demonstrated that the viability of Caco- 2 cells was significantly affected when MICU1 was silenced. Across the 10-day culturing period, the OD values for the culture of MICU1-KD colonic epithelial cells were significantly

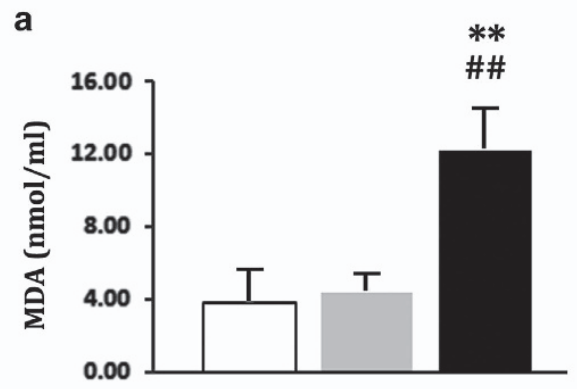

\section{Control}

NC

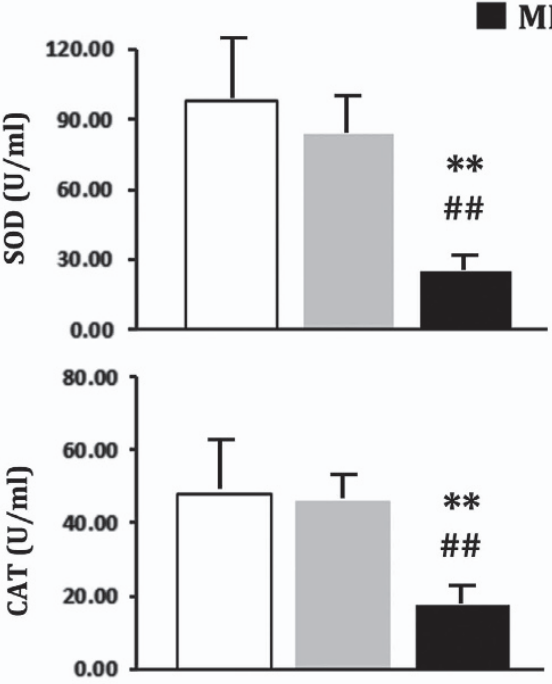

MICU1 KD

b
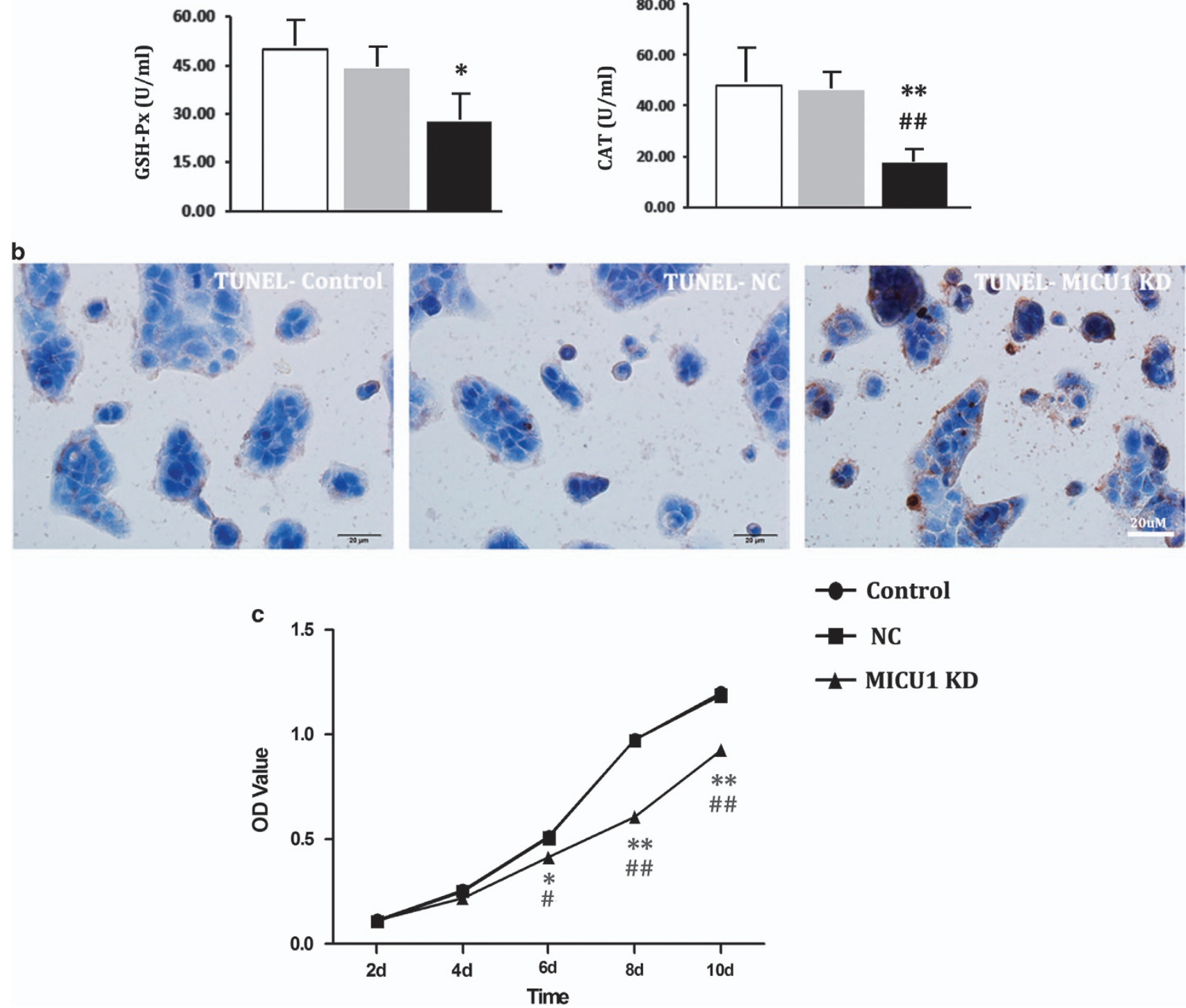

$\rightarrow$ Control

$\rightarrow$ NC

$\leftarrow$ MICU1 KD

Figure 5. MICU1 regulates the oxidative responses, apoptosis and cell growth in vitro. (a) Detection of oxidative imbalance. In MICU1-KD cells, the oxidative product MDA was significantly increased, whereas the antioxidant products (GSH-Px, CAT, and SOD) decreased. (b) TUNEL stain. The nuclei of the apoptotic cells were positively stained brownish yellow. The proportion of the positively immunostained cells were significantly higher than those of NC group and control group. (c) Growth curve. From day 6, the OD values of cultured MICU1-KD cells were significantly lower than those of NC and control. Statistical analysis was performed by one-way ANOVA test and independent $t$-test. versus control, ${ }^{*} P<0.05,{ }^{* *} P<0.01$; versus NC, ${ }^{\#} P<0.05$, ${ }^{\# \#} P<0.01$. NC (negative control group), Caco-2 cells transfected with empty lentiviral vector. MICU1-KD, MICU1-knockdown. 
lower than those of the control group or the NC group (Figure $5 \mathrm{c}$ ); the OD values of the control group and the NC group matched each other.

\section{DISCUSSION}

Damage to the barrier functions of the gastrointestinal tract, triggered by $\mathrm{IAH}$-associated ischemia and subsequent oxidative injury, is considered the pathophysiological basis for the IAHassociated MOF cascade. ${ }^{9,13}$ In this study, we further explored the upstream mechanisms underlying $\mathrm{IAH}$-induced increased intestinal permeability. We found that MICU1, the gatekeeper of mitochondrial calcium ion influx, is deeply involved in the regulation of oxidative damage, by modulating the proliferation and apoptosis of colonic epithelial cells. Silencing of MICU1 destroyed Caco-2 cell barrier integrity, promoted paracellular permeability, and impaired the expression of TJ proteins. All of these alterations can be found in rats with $\mathrm{IAH}^{9}{ }^{9}$ In line with the findings in cultured cells, a compensatory increase in MICU1 was detected in rats with IAH. All of these results suggest that MICU1 is essential for regulating intestinal barrier function. We conclude that disturbances in MICU1 levels are involved in the increased intestinal permeability induced by IAH.

MICU1 is also called CBARA1 (calcium binding atopy-related autoantigen 1). It has recently be demonstrated that it functions as a gatekeeper that sets the $\mathrm{Ca}^{2+}$ threshold for $\mathrm{Ca}^{2+}$ uptake by MCU, to regulate oxidative stress, both in vivo and in vitro. ${ }^{10,12,14}$ As this gatekeeper function was identified, a considerable number of studies investigated the expression and function of MICU1 in undifferentiated human embryonic stem cells (hESCs), hepatocytes, dermal endothelial cells, HeLa cells, breast epithelia, and pancreatic $\beta$-cells, and other cell types. ${ }^{15-21}$ Nevertheless, before our investigations, few studies had focused on the expression of MICU1 in enterocytes or its involvement in IAH-induced intestinal barrier damage. In fact, mitochondrial dysfunction has been confirmed in the development of IAH, surgical stress, and other similar conditions. Exposure to increased IAP is reported to lead to mitochondrial swelling and discontinuous intercellular tight junctions. $^{3}$ Surgical stress, the most common cause for $\mid \mathrm{AH}$, results in alterations to mitochondrial respiration and the thiol redox status of enterocytes. These changes were associated with altered mitochondrial matrix enzyme activity, decreased superoxide dismutase activity, induction of the mitochondrial permeability transition, as well as impairments in the $\mathrm{Ca}_{\mathrm{m}}^{2+}$ flux. ${ }^{22}$ Meanwhile, previous studies have elucidated that intestinal oxidative stress is responsible for the altered intestinal permeability seen during surgical stress, or the development of $\mathrm{IAH}_{.}{ }^{9,23}$ Thus, it is not difficult to understand how the intestinal barrier dysfunction that develops under IAH, or surgical stress, is related to disturbances in MICU1 levels. This is because MICU1 is involved in oxidative regulation, as we have confirmed in our studies.

MICU1 has been determined to be vital for liver tissue repair and hESC self-renewal and pluripotency. ${ }^{15,16}$ Antony et al. found that on liver-specific MICU1 loss, partial hepatectomy resulted in lethargic mice. Furthermore, the pro-inflammatory phase does not resolve in such mice and liver regeneration fails, with evidence of impaired cell cycle entry and extensive necrosis. Preventing the opening of the mitochondrial permeability transition pore (mPTP) in MICU1-knockdown mice successfully rescued hepatocyte proliferation. ${ }^{15}$ Moreover, MICU1-knockdown in hESCs attenuated cell growth and produced $G_{0} / G_{1}$-phase cell cycle arrest, indicating that MICU1 has a role in maintaining stemness, cell cycle progression, and proliferation. ${ }^{18}$ Our results are consistent with these findings. As shown in Table 1, MICU1 struggled to antagonize the oxidative stress, induced by a 90-min exposure to $\mathrm{IAH}$, by increasing its transcriptional expression. It should be noted, however, that no obvious changes were detected in the levels of MICU1. Studies with micu1 knocked-down enterocytes further demonstrated that MICU1 protects tissues by regulating oxidative stress and cellular proliferation. As shown in Figure 5, without micu1, the apoptotic rate of Caco-2 cells increased, whereas cellular viability was lowered; this was accompanied by an oxidative imbalance. All of these changes were responsible for the disrupted TJ permeability and paracellular transport.

The gut is the most sensitive to increased IAP. We previously reported that even mild increase in IAP $(8-12 \mathrm{mmHg})$ is accompanied by slight mucosal damage and an oxidative imbalance. ${ }^{9}$ Our findings have been supported by the work of Maddison et al. ${ }^{24}$ We found that as the IAP increases to $20 \mathrm{~mm} \mathrm{Hg}$ for $90 \mathrm{~min}$, intestinal barriers become seriously disrupted and dysbiosis occurs. ${ }^{25}$ However, in the present study, at the same level of IAH, MICU1 exhibited a compensatory response towards the increased intestinal permeability, indicating that under $I A H$, the gut promotes a self-protective mechanism. Further studies would be needed to establish whether MICU1 would be disrupted as the course/degree of IAH progresses, and to determine what roles are played by MICU1 in the subsequent abdominal compartment syndrome and gut-derived sepsis.

\section{CONCLUSIONS}

The current study is the first to identify the MICU1-related upstream mechanism for IAH-induced oxidative imbalances, and the subsequent increase in the intestinal permeability. MICU1 may be a promising target for intervening in $\mathrm{IAH}$-induced gut-derived sepsis and MOF. Because MICU1 has a key role in maintaining intestinal barrier integrity by regulating oxidative stress and cellular proliferation.

\section{MATERIALS AND METHODS}

Animal studies

Establishment of IAH-induced increased intestinal permeability in rat models. To explore the involvement of MCU/MICU1 in IAH-induced intestinal permeability changes, 8 male SPF Sprague-Dawley rats (8-weeks-old, weight: $200-250 \mathrm{~g}$ ) were randomly assigned to the 'IAH group' $(20 \mathrm{mmHg}$, $n=4)$, or to the control group $(n=4)$. The acute IAH animal model $(20 \mathrm{mmHg}$ ) was established using a 90-min nitrogen pneumoperitoneum procedure. Increased intestinal permeability was identified by the detection of intestinal permeability to FITC-dextran (FD-4, molecular weight: 4000 Da; Sigma-Aldrich, St. Louis, MO, USA, Product No. 68059) as previously described. ${ }^{9}$ Briefly, following anesthesia by intraperitoneal injection of sodium pentobarbital $(40 \mathrm{mg} / \mathrm{kg}$ ), nitrogen pneumoperitoneum was created by a disposable venous infusion needle connected to a micro-infusion pump. The micro-infusion pump was linked to a blood pressure meter to dynamically monitor the IAP. After $90 \mathrm{~min}$, a $10-\mathrm{cm}$ segment of the distal ileum with preserved superior mesenteric vessels was dissected $3 \mathrm{~cm}$ proximal to the cecum. One milliliter of phosphatebuffered saline (PBS; $0.1 \mathrm{~mol} / \mathrm{l}, \mathrm{pH} 7.2$ ) containing $25 \mathrm{mg}$ of FD-4 was injected into this ligated $10-\mathrm{cm}$ segment of intestinal lumen, and the lumen was carefully replaced in the abdomen, covered, and protected with gauze soaked in warm saline. Portal venous blood samples were collected to analyze the FD-4 concentrations spectrophotometrically, after $30 \mathrm{~min}$, at an excitation wavelength of $492 \mathrm{~nm}$ and an emission wavelength of $518 \mathrm{~nm}$. The colonic tissues were frozen by immersion in liquid nitrogen and stored at $-80^{\circ} \mathrm{C}$, until needed for further experiments. For the control group, nitrogen was not injected.

All experimental procedures, including the care and handling of animals, were performed following international guidelines (Guide for the Care and Use of Laboratory Animals, Institute of Laboratory Animal Resources, Commission on Life Sciences, National Research Council, National Academy Press, Washington, DC, USA, 1996). ${ }^{26}$ The rationale, design, and protocols for our study were approved by the Peking University Biomedical Ethics Committee-Experimental Animal Ethics Branch (Approval No. LA2013-12), before the initiation of experiments. The rats were housed solitarily in polypropylene cages and kept under standard controlled environmental conditions with $12 \mathrm{~h}$ light/dark cycles. The rats had free access to standard rat chow and water, which were autoclaved before use. 
The rats were deprived of food, but not water, for $12 \mathrm{~h}$ before inducing nitrogen pneumoperitoneum for 90 min (described below).

All surgeries were conducted under sodium pentobarbital anesthesia (intraperitoneal injection, $40 \mathrm{mg} / \mathrm{kg}$ ) and all efforts were made to minimize pain. After the procedure was complete, the animals were euthanized using an overdose of sodium pentobarbital (intraperitoneal injection, $160 \mathrm{mg} / \mathrm{kg})^{9}{ }^{9}$

Changes in the expression of MICU1/MCU in rats with $\mathrm{IAH}$ Western blotting. Standard western blotting analyses of MICU1 and MCU were conducted as previously described. ${ }^{9}$ Colonic tissue samples (or cultured cells) were homogenized in lysis buffer $(20 \mathrm{mmol} / \mathrm{I}$ Tris- $\mathrm{HCl}(\mathrm{pH}$ 7.5), $1 \%$ Triton X-100, $0.2 \mathrm{~mol} / \mathrm{l} \mathrm{NaCl}, 2 \mathrm{mmol} / \mathrm{l}$ ethylenediaminetetraacetic acid, $2 \mathrm{mmol} / \mathrm{l}$ ethylene glycol tetraacetic acid, $1 \mathrm{~mol} / \mathrm{l}$ dithiothreitol, and $2 \mathrm{~mol} / \mathrm{l}$ aprotinin). Proteins $(60 \mu \mathrm{g})$ were electrophoresed using SDS-PAGE (8\%) and transferred to a polyvinylidene fluoride membrane. The membranes were blocked for $1 \mathrm{~h}$ with nonfat dried milk in Trisbuffered saline containing $0.05 \%$ Tween-20 (TTBS) at room temperature. They were then incubated overnight, with gentle shaking, at $4{ }^{\circ} \mathrm{C}$ with antibodies against MCU and MICU1. (Details for all the primary antibodies that we applied are provided in Table 3). Thereafter, goat antirabbit fluorescently labeled secondary antibodies (1:10 000; LI-COR Biosciences, Lincoln, NE, USA) were added for $1 \mathrm{~h}$ to the membranes at room temperature. Bound proteins were visualized following scanning of the membranes with an Odyssey Infrared Imaging System (LI-COR Biosciences, Lincoln, NE, USA).

Quantitative reverse transcription $P C R$ analysis. Total RNA was isolated from colonic tissue (or Caco-2 cells), using TRIzol Reagent (Invitrogen, Carlsbad, CA, USA), according to the manufacturer's instructions. RNA samples were then standardized and reverse transcribed using a Transcriptor First Strand cDNA Synthesis Kit (Roche, Basel, Switzerland) with an oligo-dT/random nonamer primer mixture. RT-qPCR was carried out using a 'Roche FS Universal SYBR Green Master' mixture (Roche), according to the manufacturer's instructions, and a 'Viia7' system (Applied Biosystems, Shanghai, China).

Thermal cycling parameters: Started with $10 \mathrm{~min}$ at $95^{\circ} \mathrm{C}$, followed by 40 cycles of $95^{\circ} \mathrm{C}$ for $15 \mathrm{~s}, 60^{\circ} \mathrm{C}$ for $30 \mathrm{~s}$, and $72{ }^{\circ} \mathrm{C}$ for $30 \mathrm{~s}$. The specificity of the $\mathrm{PCR}$ products was verified by melting curve analysis. Specific primers for both the genes, Mcu (NM_001106398.1, 1364 bp) and Micu1 (NM 199412.1, 2300 bp) were designed by Qiyin Biotechnology (Shanghai, China). The primer sequences were:

- $M c u$-F: 5'-ACCCTGAACGATGTGAAGA-3

- McU -R: 5'-CTCCGCTTTCCTGCTAAT-3'

- Micu1-F: 5'-CAAGTCTGGCTTATGTTCG-3'

- Micu1-R: 5'-AGATTCTCCCGTCTACCG-3'

\section{CELL STUDIES}

Cell culture

Caco-2 cells were cultured at $37{ }^{\circ} \mathrm{C}$ under a $5 \% \mathrm{CO}_{2}$ humidifiedatmosphere in DMEM, supplemented with $10 \%$ fetal bovine serum, $1 \%$ nonessential amino acids, $100 \mathrm{U} / \mathrm{ml}$ penicillin, and $100 \mu \mathrm{g} / \mathrm{ml}$ streptomycin. Cells were used in experiments $\sim 14$ days after achieving confluence, to allow for their differentiation into intestinal epithelial cells. The medium was replaced every 3 days.
Generation of stable MICU1-knockdown cell lines

We generated stable clones using lentiviral shRNAs targeting different regions of the micu1 gene (Table 2). Four different lentiviruses, each carrying shRNAs targeting different regions of micu1, were produced by co-transfecting 293T cells with MICU1 lentiviral shRNA constructs (KeyGEN BioTECH, Jiangsu, China; Table 2), psPAX2, and pMD2.G (Addgene, Cambridge, MA, USA), as previously described. ${ }^{10,27}$ Caco-2 cells $\left(5 \times 10^{5}\right.$ per well) grown in 6-well plates were transduced with MICU1 lentiviruses, selected with puromycin $(2 \mu \mathrm{g} / \mathrm{ml}) 72 \mathrm{~h}$ post-transduction for 6-10 days, and then expanded. Knockdown was assessed by western blotting.

Influence of MICU1 on epithelial-barrier function in cultured Caco2 cells

TEER and detection of transport using FSA. To analyze the effect of MICU1 on paracellular permeability, a monolayer of Caco-2 cells was established and measured as TEER using a Millicell-ERS device (Millipore, Bedford, MA, USA), as previously described. ${ }^{28}$ Briefly, $2 \times 10^{5}$ cells $/ \mathrm{ml}$ were seeded on Transwell permeable supports and cultured. Then, TEER was measured every day and calculated as:

TEER $=\left(R_{\mathrm{m}}-R_{\mathrm{i}}\right) \times S$

( $R_{\mathrm{m}}$, transmembrane resistance; $R_{\mathrm{i}}$, intrinsic resistance of a cell-free medium; and $S$, the surface area of the membrane in $\mathrm{cm}^{2}$, $\left.1.12 \mathrm{~cm}^{2}\right)$.

When the TEER value exceeded $250 \Omega \mathrm{cm}^{2}$, paracellular transport through Caco-2 cell monolayers was determined by measuring the apical-to-basolateral clearance of fluorescein (FSA, sodium salt) (Sigma-Aldrich, St Louis, MO, USA, Lot. F6377), as previously described. ${ }^{29}$ Briefly, once the TEER values had been determined, the medium in all compartments was replaced with Hank's balanced salt solution; FSA was then added to the apical compartment $(10 \mathrm{mg} / \mathrm{l}$ in $200 \mu \mathrm{l})$. One hour after the addition of FSA, $100 \mu \mathrm{l}$ solution was taken from the basolateral compartment and the fluorescence was measured $\left(\lambda_{\text {ex }}: 428 \mathrm{~nm}\right.$; $\left.\lambda_{\mathrm{em}}: 536 \mathrm{~nm}\right)$. FSA clearance $\left(\mathrm{CL}_{\mathrm{FSA}}\right)$ was calculated using the equation:

$\mathrm{CL}_{\mathrm{FSA}}=F_{\mathrm{ab}} /\left(F_{\mathrm{FSA}} \times S\right)$; where ' $F_{\mathrm{ab}}$ ' represents the flux of FSA in fluorescence units/ $h$; ' $F_{\mathrm{FSA}}$ ', the fluorescence of FSA in the apical compartment; and ' $S$ ', the surface area of the membrane in $\mathrm{cm}^{2}$.

Immunofluorescence analysis of tight junction proteins (TJs). To explore the association between the expression of MICU1 and TJs, standard immunofluorescence and western blotting were performed in Caco-2 cells (control) and MICU1-silenced cells. For immunostaining, cells were seeded onto glass coverslips in 6-well plates and fixed overnight with 4\% formaldehyde. The cells were then permeabilized with $0.1 \%$ Triton X-100 in PBS and stained with anti-claudin 1, anti-occludin, or anti-ZO-1 antibodies $\left(5 \times 10^{5}\right.$ per well). Following a PBS wash, the cells were incubated with fluorescein isothiocyanate-linked secondary antibodies, for $1 \mathrm{~h}$ at room temperature (KeyGEN BioTECH, Jiangsu, China, Lots. KGAA26 \& KGAA27). Nuclei were counterstained with

Table 3. Details of the first antibodies

\begin{tabular}{lllllll}
\hline Reagents & Claudin 1 & Occludin & GAPDH & MICU1 & ZO-1 \\
\hline Company & Abcam & Abcam & Abcam & Abcam & Abcam \\
Product number & ab15098 & ab31721 & Ab8245 & ab102830 & ab190085 \\
Source & Rabbit & Rabbit & Mouse & Rabbit & Goat & ab121499 \\
Dilution & $1: 200$ & $1: 200$ & $1: 1000$ & $1: 1000$ & Rabbit & $1: 200$ \\
\hline
\end{tabular}


4',6-diamidino-2-phenylindole. The sections were scanned by confocal microscopy (Olympus IX51, Tokyo, Japan).

The influence of MICU1 on the pro-oxidant/antioxidant balance in cultured Caco-2 cells

The influence of MICU1 on the pro-oxidant/antioxidant balance was measured by ELISA. We detected the levels of MDA (Lot. KGT003), glutathione peroxidase (GSH-Px, Lot. KGT014), catalase (CAT, Lot. KGT017), and serum superoxide dismutase (SOD, Lot. KGT00150) in cultured Caco-2 cells, according to the manufacturer's instructions (KeyGEN BioTECH, Jiangsu, China). Each test was repeated three times. Measurements were quantified by spectrophotometric recordings (Thermo Fisher Scientific, Waltham, MA, USA).

\section{TUNEL assay for apoptosis}

Apoptosis resulting from the knockdown of MICU1 detected by a terminal deoxynucleotidyl transferase dUTP nick-end labeling kit (TUNEL, KeyGEN BioTECH, Jiangsu, China, Lot. KGA702), according to the manufacturer's instructions. The percentage of TUNELpositive cells was calculated, and compared between both groups. The experiment was repeated three times. Five randomly selected fields were chosen in each section. In total, 15 datasets were recorded for each group and compared.

\section{MTT assay for cell growth}

Cultured cells were seeded at a density of $3 \times 10^{3}$ cells per well in 96 -well microtiter culture plates. After $2,4,6,8$, and 10 days of culturing, separate wells received a $20 \mu$ addition of MTT solution $(5 \mathrm{mg} / \mathrm{ml}$ in PBS; St. Louis, MO, USA); these were each incubated for a further $4 \mathrm{~h}$. On termination, the supernatant was aspirated and MTT was converted to formazan by metabolically viable cells. The formazan was then dissolved in $150 \mu \mathrm{l}$ of DMSO. The plates were mixed for 10 min on a gyratory shaker, and absorbance was measured at $490 \mathrm{~nm}$.

\section{Statistical analysis}

Statistical analyses were performed using SPSS 16.0 software (SPSS, Chicago, IL, USA). Data were expressed as mean values \pm S.D. One-way analysis-of-variance tests and independent $t$-tests were performed. $P$-values $<0.05$ were considered statistically significant.

\section{ABBREVIATIONS}

$\mathrm{Ca}_{\mathrm{m}}^{2+}$, mitochondrial $\mathrm{Ca}^{2+}$; CAT, catalase; $\mathrm{CL}_{\mathrm{FSA}}$, FSA clearance; DMSO, dimethyl sulfoxide; FD-4, FITC-dextran, 4000Da; Fab, the flux of FSA in fluorescence units; $F_{F S A}$, the fluorescence of FSA in the apical compartment; FSA, fluorescein sodium salt; GSH-Px, glutathione peroxidase; hESCs, human embryonic stem cells; IAP, intra-abdominal pressure; IAH, intra-abdominal hypertension; KD, knockdown; MCU, mitochondrial calcium uniporter; MDA, malondialdehyde; MICU1, mitochondrial $\mathrm{Ca}^{2+}$ uptake 1; MOF, multi-organ failure; MPTP, mitochondrial permeability transition pore; mROS, mitochondrial reactive oxygen species; MTT, 3-(4,5)-dimethylthiahiazo (-z-y1)-3,5-di- phenytetrazoliumromide; NC, negative shRNA control; SOD, serum superoxide dismutase; TEER, transepithelial electrical resistance; TTBS, Tris-buffered saline containing Tween20; TJ, tight junction; TUNEL, terminal deoxynucleotidyl transferase dUTP nick-end labeling.

\section{ACKNOWLEDGEMENTS}

This work was supported by the Beijing Science and Technology Plan (Z151100004015135, Z141107002514020), Natural Science Foundation of Beijing Municipality (7154251) and The National Natural Science Foundation of China (81441061)

\section{COMPETING INTERESTS}

The authors declare no conflict of interest.

\section{REFERENCES}

1 Kirkpatrick AW, Roberts DJ, De Waele J, Jaeschke R, Malbrain ML, De Keulenaer B et al. Intra-abdominal hypertension and the abdominal compartment syndrome: updated consensus definitions and clinical practice guidelines from the World Society of the Abdominal Compartment Syndrome. Intensive Care Med 2013; 39: 1190-1206.

2 Kaussen T, Srinivasan PK, Afify M, Herweg C, Tolba R, Conze J et al. Influence of two different levels of intra-abdominal hypertension on bacterial translocation in a porcine model. Ann Intensive Care 2012; 2: S17.

3 Cheng J, Wei Z, Liu X, Li X, Yuan Z, Zheng J et al. The role of intestinal mucosa injury induced by intra-abdominal hypertension in the development of abdominal compartment syndrome and multiple organ dysfunction syndrome. Crit Care 2013; 17: R283.

4 Sukhotnik I, Bejar J, Srugo I, Krausz MM, Bernshteyn A, Hirsh M et al. Adverse effects of increased intra-abdominal pressure on small bowel structure and bacterial translocation in the rat. J Laparoendosc Adv Surg Tech A 2006; 16 404-410.

5 Gong G, Wang P, Ding W, Zhao Y, Li J. Microscopic and ultrastructural changes of the intestine in abdominal compartment syndrome. J Invest Surg 2009; 22: 362-367.

6 Kotidis E, Papavramidis T, loannidis K, Koliakos G, Lazou T, Cheva A et al. Can chronic intra-abdominal hypertension cause oxidative stress to the abdominal wall muscles? An experimental study. J Surg Res 2012; 176: 102-107.

7 Gong G, Wang P, Ding W, Zhao Y, Li J. The role of oxygen-free radical in the apoptosis of enterocytes and bacterial translocation in abdominal compartment syndrome. Free Radic Res 2009; 43: 470-477.

8 Eleftheriadis E, Kotzampassi K, Papanotas K, Heliadis N, Sarris K. Gut ischemia, oxidative stress, and bacterial translocation in elevated abdominal pressure in rats. World J Surg 1996; 20: 11-16.

9 Leng Y, Zhang K, Fan J, Yi M, Ge Q, Chen L et al. Effect of acute, slightly increased intra-abdominal pressure on intestinal permeability and oxidative stress in a rat model. PLoS One 2014; 9: e109350.

10 Mallilankaraman K, Doonan P, Cárdenas C, Chandramoorthy HC, Müller M, Miller R et al. MICU1 is an essential gatekeeper for MCU-mediated mitochondrial $\mathrm{Ca}(2+)$ uptake that regulates cell survival. Cell 2012; 151: 630-644.

11 Perocchi F, Gohil VM, Girgis HS, Bao XR, McCombs JE, Palmer AE et al. MICU1 encodes a mitochondrial $\mathrm{EF}$ hand protein required for $\mathrm{Ca}(2+)$ uptake. Nature 2010; 467: 291-296.

12 Csordás G, Golenár T, Seifert EL, Kamer KJ, Sancak Y, Perocchi F et al. MICU1 controls both the threshold and cooperative activation of the mictochondrial $\mathrm{Ca}^{2+}$ uniporter. Cell Metab 2013; 17: 976-987.

13 Sánchez-Miralles A, Castellanos G, Badenes R, Conejero R. Abdominal compartment syndrome and acute intestinal distress syndrome. Med Intensiva 2013; 37: 99-109.

14 Liu JC, Liu J, Holmström KM, Menazza S, Parks RJ, Fergusson MM et al. MICU1 serves as a molecular gatekeeper to prevent in vivo mitochondrial calcium overload. Cell Rep 2016; 16: 1561-1573.

15 Antony AN, Paillard M, Moffat C, Juskeviciute E, Correnti J, Bolon B et al. MICU1 regulation of mitochondrial $\mathrm{Ca}(2+)$ uptake dictates survival and tissue regeneration. Nat Commun 2016; 7: 10955.

16 Chen K, Hsu LT, Wu CY, Chang SY, Huang HT, Chen W. CBARA1 plays a role in stemness and proliferation of human embryonic stem cells. PLoS One 2013; 8 : e63653.

17 De Stefani D, Raffaello A, Teardo E, Szabò I, Rizzuto R. A forty-kilodalton protein of the inner membrane is the mitochondrial calcium uniporter. Nature 2011; 476: 336-340.

18 Aichberger KJ, Mittermann I, Reininger R, Seiberler S, Swoboda I, Spitzauer S et al. Hom s 4, an IgE-reactive autoantigen belonging to a new subfamily of calciumbinding proteins, can induce Th cell type 1-mediated autoreactivity. J Immunol 2005; 175: 1286-1294.

19 Perocchi F, Gohil VM, Girgis HS, Bao XR, McCombs JE, Palmer A et al. MICU1 encodes a mitochondrial $\mathrm{EF}$ hand protein required for $\mathrm{Ca}(2+)$ uptake. Nature 2010; 467: 291-296.

20 Alam MR, Groschner LN, Parichatikanond W, Kuo L, Bondarenko Al, Rost R et al. Mitochondrial $\mathrm{Ca} 2+$ uptake 1 (MICU1) and mitochondrial Ca2+ uniporter (MCU) contribute to metabolism-secretion coupling in clonal pancreatic $\beta$-cells. J Biol Chem 2012; 287: 34445-34454.

21 Hall DD, Wu Y, Domann FE, Spitz DR, Anderson ME. Mitochondrial calcium uniporter activity is dispensable for MDA-MB-231 breast carcinoma cellsurvival. PLoS One 2014; 9: e96866. 
22 Ramachandran A, Patra S, Balasubramanian KA. Intestinal mitochondrial dysfunction in surgical stress. J Surg Res 2001; 99: 120-128.

23 Anup R, Aparna V, Pulimood A, Balasubramanian KA. Surgical stress and the small intestine: role of oxygen free radicals. Surgery 1999; 125: 560-569.

24 Maddison L, Starkopf J, Reintam Blaser A. Mild to moderate intra-abdominal hypertension: does it matter? World J Crit Care Med 2016; 5: 96-102.

25 Leng Y, Yi M, Fan J, Bai Y, Ge Q, Yao G. Effects of acute intra-abdominal hypertension on multiple intestinal barrier functions in rats. Sci Rep 2016; 6: 22814

$26 \mathrm{NIH}$. Guide for the Care and Use of Laboratory Animals. The National Academies Press: Washington, DC, USA: 1996.

27 Naldini L, Blomer U, Gallay P, Ory D, Mulligan R, Gage FH et al. In vivo gene delivery and stable transduction of nondividing cells by a lentiviral vector. Science 1996; 272: 263-267.

28 Contreras TC, Ricciardi E, Cremonini E, Oteiza PI. (-)-Epicatechin in the prevention of tumor necrosis alpha-induced loss of Caco-2 cell barrier integrity. Arch Biochem Biophys 2015; 573: 84-91.
29 Unno N, Menconi MJ, Salzman AL, Smith M, Hagen S, Ge Y et al. Hyperpermeability and ATP depletion induced by chronic hypoxia or glycolytic inhibition in Caco-2BBe monolayers. Am J Physiol 1996; 270: G1010-G1021.

(c) This work is licensed under a Creative Commons Attribution 4.0 International License. The images or other third party material in this article are included in the article's Creative Commons license, unless indicated otherwise in the credit line; if the material is not included under the Creative Commons license, users will need to obtain permission from the license holder to reproduce the material. To view a copy of this license, visit http://creativecommons.org/licenses/ by/4.0/

(c) The Author(s) 2016 